\title{
Telomere dynamics in relation to experimentally increased locomotion costs and fitness in great tits
}

\author{
Els Atema ${ }^{1}$, Arie van Noordwijk ${ }^{2}$, and Simon Verhulst ${ }^{3}$ \\ ${ }^{1}$ Nederlands Instituut voor Ecologie \\ ${ }^{2}$ Netherlands Institute of Ecology \\ ${ }^{3}$ University of Groningen
}

May 7, 2021

\begin{abstract}
Evidence that telomere length (TL) and dynamics can be interpreted as proxy for 'life stress' experienced by individuals stems largely from correlational studies. We tested for effects of an experimental increase of workload on telomere dynamics by equipping male great tits (Parus major) with a 0.9 gram backpack for a full year. In addition, we analysed associations between natural life-history variation, TL and TL dynamics. Carrying $5 \%$ extra weight for a year did not significantly accelerate telomere attrition. This agrees with our earlier finding that this experiment did not affect survival or future reproduction. Apparently, great tit males were able to compensate behaviourally or physiologically for the increase in locomotion costs we imposed. We found no cross-sectional association between reproductive success and TL, but individuals with higher reproductive success (number of recruits) lost fewer telomere base pairs in the subsequent year. We used the TRF method to measure TL, which method yields a TL distribution for each sample, and the association between reproductive success and telomere loss was more pronounced in the higher percentiles of the telomere distribution, in agreement with the higher impact of ageing on longer telomeres within individuals. Individuals with longer telomeres and less telomere shortening were more likely to survive to the next breeding season, but these patterns did not reach statistical significance. Whether successful individuals are characterized by losing fewer or more base pairs from their telomeres varies between species, and we discuss aspects of ecology and social organisation that may explain this variation.
\end{abstract}

\section{Hosted file}

Atema et al - Great tit telomeres.pdf available at https://authorea.com/users/412454/ articles/521164-telomere-dynamics-in-relation-to-experimentally-increased-locomotioncosts-and-fitness-in-great-tits 